\title{
Nonstandard mathematical model for fatigue failure
}

\author{
Petru CARDEI ${ }^{1, \mathbf{a}^{*}}$, Gheorghe VOICU ${ }^{2, \mathbf{b}}$, Mihai MATACHE ${ }^{3, \mathrm{c}}$, Iulian VOICEA ${ }^{4, \mathrm{~d}}$, \\ Vergil MURARU ${ }^{5, e}$ and Mihai LUDIG ${ }^{6, f}$ \\ ${ }^{1,3,4,5,6}$ National Institute of Research-Development for Machines and Installations Designed to \\ Agriculture and Food Industry - INMA Bucharest, Romania \\ ${ }^{2}$ Department of Biotechnical Systems, University POLITEHNICA of Bucharest, Romania \\ apetru_cardei@yahoo.com, bghvoicu_2005@yahoo.com, cgabimatache@yahoo.com, \\ dvoicea_iulian@yahoo.com, ${ }^{e}$ virgil.muraru@gmail.com,lud.mihai@yahoo.com
}

Keywords: fatigue, nonstandard, simulation, model.

\begin{abstract}
Tests performed under simulated regime, use simulating tools with different structures and often have a corresponding physical test to complete certain conclusions in order to explain complex phenomena that are difficult, impossible, or very costly to run physically. Computer simulations are also called virtual tests beside which there are mechanical or small scale analog simulators. Among the virtual tests for mechanical structures there are structural analysis tests that use numerical analysis methods. Sometimes, in order to understand the phenomena that occurs in the material structure, new complex theoretical models were used for explaining the material quality change. One such application is proposed, which incorporates an old author's model, for the fatigue and quality phenomenon study of the material change.
\end{abstract}

\section{Introduction}

According to [7] and [8] when applying variable loads on a structure, the material of which is made manifests a sensitive decrease of resistance characteristics compared with the same static characteristics. This phenomenon is called fatigue. The mechanical characteristics of the definition are called fatigue limit or fatigue, see [7], [8].

The most widely used mathematical model of fatigue for metallic materials is introduced in [9] by August Wohler. For this mathematical model the fatigue limit is the stress level below which component should have infinite life or exceeding it, notifies fatigue damage. A number of researchers have deepened Wohler' theory, developing a complex model of the metal fatigue phenomenon: Bauschinger, Gerber and Goodman, for example, [10], [11], [12]. Thus a theoretical and empirically model of the metal fatigue phenomenon was completed. This model is a descriptive (phenomenological) mathematical model. At the beginning of the 20th century, J. A. Ewing demonstrated that the origin of the fatigue failure consist in microscopic cracks - triggering and propagation. Alan A. Griffith founded the fracture mechanics in 1920, investigating cracks in brittle glass. Ewing and Griffith proposed models based on phenomena taking place in the microstructure of materials. These are structural models.

The main problem exposed in this article is finding an integral-differential mathematical model of the material fatigue phenomenon. This model is based on continuum mechanics. The construction method of such a model is presented in [2]. Unlike [2], based on experimental facts, the model presented in this article chooses as fatigue accumulator operator the cross-sectional area of the straight bar. In short, the proposed model explains fatigue by reducing the cross sectional area of a beam (with random or specified locations by the presence of a stress concentrator).

It shows the operation of the model, namely the response of the bolt to the cyclic traction load. It also presents the method by which the model can generate a durability diagram for material. The parameters of the model, which facilitates the approximation of the experimental diagram durability will be specified. In the proposed model, these parameters distinguish the material. A diagram of 
sustainability is not to be considered in this article, but the authors will makes such attempt in the next articles.

\section{Materials and experimental procedure}

\section{Experimental facts underlying the mathematical model}

The authors of this paper have dealt with various tests of mechanical structures over time. Most often physical tests at real scale were used, but often these were supplemented by numerical experiments (simulated tests) and accelerated testing. Elementary structures tried to fatigue presents a classic shape of the cross section for failure by fatigue. For example, a bolt destined for use in very high safety conditions, is broken during a fatigue test and is shown in fig. 1, 2 and 3.

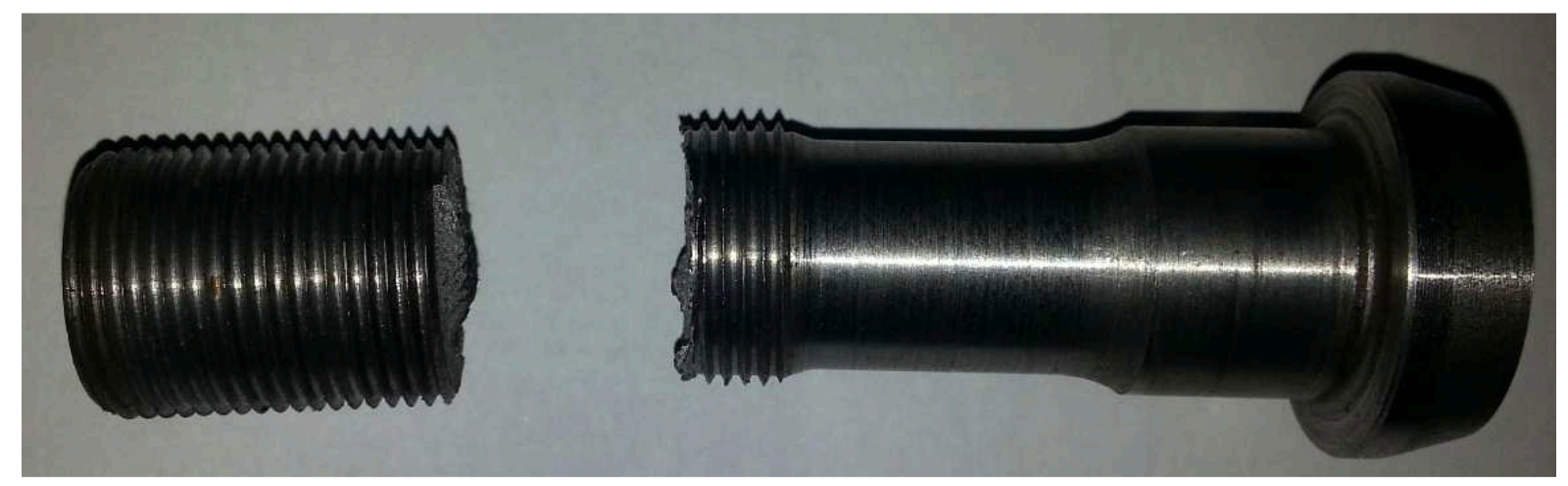

Fig. 1 Bolt broken through fatigue, side view

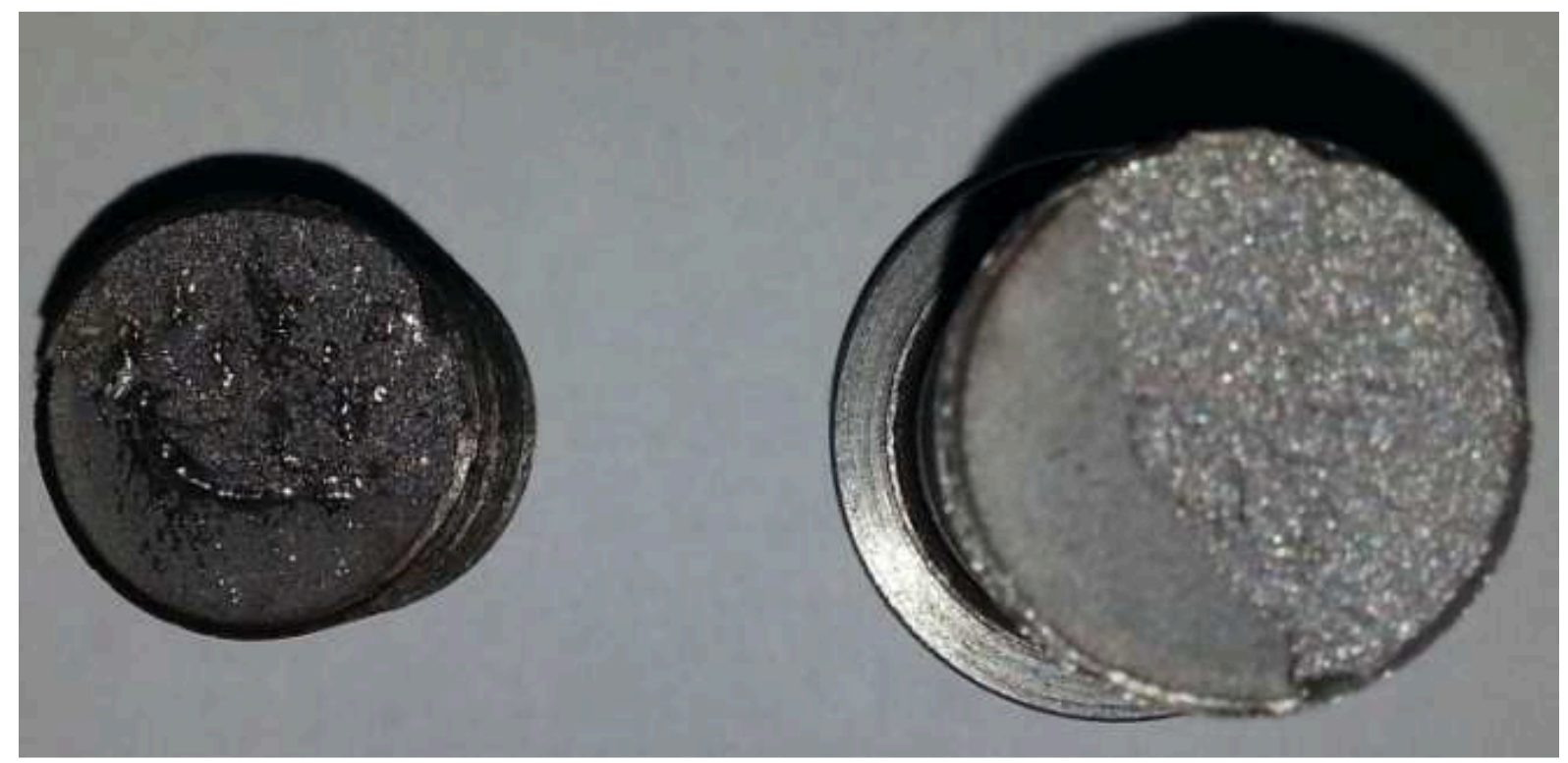

Fig. 2 The cross section of a bolt broken through fatigu

The breaking mechanism is well known [8]. The shiny part of the failure cross section corresponds to the progressive development of micro cracks. The grain cross-section corresponds to sudden rupture. The material used in these experiments was steel $16 \mathrm{NiCr} 4$, [5]. This failure mechanism suggests that the characteristic resistance diminishes with the fatigue phenomenon in the proposed mathematical model. This happens in the entire cross-sectional area of the bolt. More accurate, it is assumed that in the cross section, the gradually breaking cracks appear until the remaining non-cracked transversal sectional area has a minimum size too low for withstanding to the high tensile stress that is required for the test. 


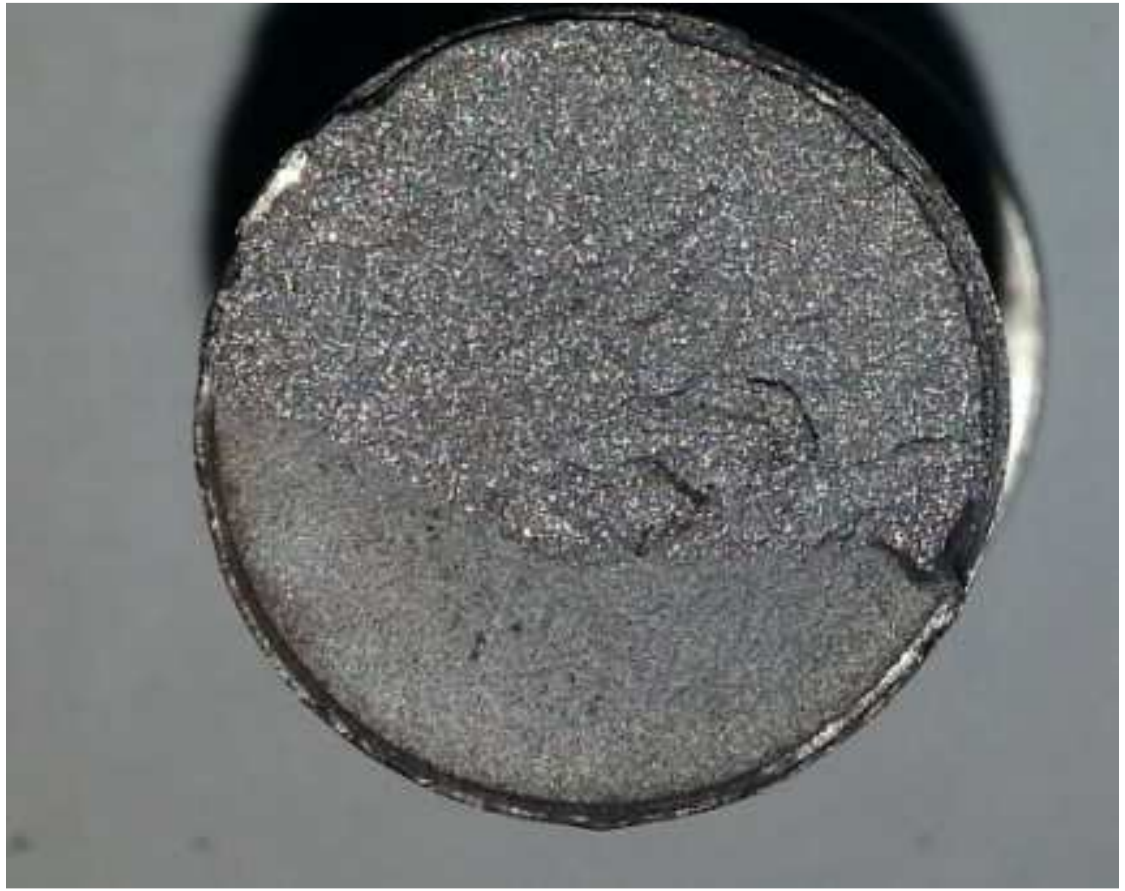

Fig. 3 Bolt broken through fatigue, breaking cross section

\section{Mathematical model for the fatigue failure}

The mathematical model from which we start is the straight one-dimensional bar in vibration, after [1]. According to [1], longitudinal oscillations of a straight bar (the one dimensional model of the straight bar subjected to an oscillating stress) is described by the equation:

$$
\frac{\partial}{\partial x}\left(A E \frac{\partial u}{\partial x}\right)=\rho A \frac{\partial^{2} u}{\partial t^{2}}
$$

with the initial condition:

$$
u(0, x)=0, \frac{\partial u}{\partial t}(0, x)=0
$$

and the boundary conditions:

$$
u(t, 0)=0, E A \frac{\partial u}{\partial x}(t, l)=F_{0} \sin \omega t
$$

\section{The modified mathematical model}

The mathematical model (1), (2), (3) is modified so that it can simulate the physical fatigue. The changing process is similar to [2], but the parameter will vary in resistance, in a different way from that of [2]. It accepts the hypothesis that the new model has a physical fatigue limit that is lower than the loading elastic limit stress, i.e.:

$$
\sigma_{R}<\sigma_{e}, \varepsilon_{R}<\varepsilon_{e}
$$

It is assumed that the cross section of the bar is constant over the entire length, with constant area $A_{0}$ at the initial time $t_{0}=0$. The basic idea of the model is that there is a section (and possibly more) in the bar which reduces its effective area for each cycle if the stress and / or strain exceeds beyond the limits of fatigue.

$$
A(t, x)=A_{0}-a \int_{0}^{s} w(s, x) d s
$$


where $w$ is a function that notifies overcoming fatigue limit and the amount that it is done. The integral operator takes into account all exceeding and it translates into loss of effective area, i.e. cross-sectional area shrinks every time when it exceeds the fatigue limit of the material. For the function $w$ there are many possible possibilities. One of these is used in [2]:

$$
w(t, x)=|| \sigma(t, x)\left|-\sigma_{R}\right| \cdot|| \varepsilon(t, x)\left|-\varepsilon_{R}\right| \cdot \Theta\left(\left(|\sigma(t, x)|-\sigma_{R}\right)\right) \cdot \Theta\left(|\varepsilon(t, x)|-\varepsilon_{R}\right)
$$

Assuming the oscillating compression stretch runs in the linear-elastic domain of the bar material, Hooke's law remains valid and the relation (6) becomes:

$$
w(t, x)=E|| \varepsilon(t, x)\left|-\varepsilon_{R}\right|^{2}=E|| \frac{\partial u}{\partial x}(t, x)\left|-\varepsilon_{R}\right|^{2} \cdot \Theta\left(|\varepsilon(t, x)|-\varepsilon_{R}\right)
$$

In the following function, we will adopt this assumption, which means that traction is small enough so that the linear elastic limit is not exceeded. The factor $a$, which multiplies the integral in (5) has the physical dimension $\mathrm{M}^{-1} \mathrm{~L}^{3} \mathrm{~T}$, that can be interpreted as, for example, the inverse of the product between a frequency and density of the bar. Taking into account all the assumptions stated, the equation which describes the bar deformation becomes:

$$
A(t, x)=A_{0}-\frac{\varphi(x) E}{v \rho} \int_{0}^{t}|| \frac{\partial u}{\partial x}(s, x)\left|-\varepsilon_{R}\right|^{2} \cdot \Theta\left(|\varepsilon(s, x)|-\varepsilon_{R}\right) d s
$$

where the dimensionless factor $\varphi(x)$ depends only on the spatial variable $x$ and, for $x=l$, becomes zero. Taking into account that:

$$
\varepsilon(t, x)=\frac{\partial u(t, x)}{\partial x}
$$

the function $A$ defined by (8) can be written in the simplest form:

$$
A(t, x)=A_{0}-\frac{\varphi(x) E}{v \rho} \int_{0}^{t}|| \varepsilon(s, x)\left|-\varepsilon_{R}\right|^{2} \cdot \Theta\left(|\varepsilon(s, x)|-\varepsilon_{R}\right) d s
$$

The function (7), which appears in [2], is called degenerative nucleus because it senses functioning in material fatigue regime and measures the loss of effective cross section of the bar. Version (6) of the degenerative nucleus, is continuous but not differentiable in relation to the spatial and temporal variable. Because of this, the partial differential equation could not be solved and was transformed into an integral - differential equation with partial derivatives, which do not contain derivatives of degenerative nucleus. However, even in these conditions, this type of degenerative nucleus leads to pronounced instability of the function that gives the development of the critical section (which will break), not only for long-time solutions, but also for the solution of the problem for a time of the order of two or three periods of traction. To improve the stability of the integral term from equation (5), we examined a continuous and differentiable function form for the degenerative nucleus. There are many such possible models. We chose one exponential form:

$$
w(t, x)=\varepsilon_{R}^{n}\left[1-e^{-\frac{\varepsilon^{2}(t, x)}{2 \varepsilon_{R}^{2}}}\right]
$$

The parameter $n$ can be used to increase the model fidelity for fatigue accumulation. 


\section{Results and discussions}

A weak (generalized) solution of the problem

The calculation of an exact or approximate solutions to problem (1) - (3), where the variation of cross-sectional area is given by formulas (8) and (10), an open question still remains. We have no proof for the existence and uniqueness of the solutions to this problem. For these reasons, we will try a weak solution in the sense of [3] or [4], for example. The problem (1) - (3), where function $A$ is given by (8) or (10) leads to a complex problem: an integral differential equation, or an integral differential equation with partial derivatives, systems rarely addressed in the literature. Moreover, inserting (10) in (1), the differential equation (1) cannot be strictly solved, because function $A$ is not differentiable with respect to the spatial variable $x$.

Solving the problem in a generalized sense, however, is done by integrating (1) equation with respect to $x$ over the entire length of the bar. Thus we obtain the equation:

$$
E A(t, l) \frac{\partial u(t, l)}{\partial x}-E A(t, 0) \frac{\partial u(t, 0)}{\partial x}=\int_{0}^{l} \rho A(t, x) \frac{\partial^{2} u(t, x)}{\partial x^{2}} d x
$$

For obtaining a weak solution in the sense of [3] or [4], the problem starts with a home solution of the form:

$$
u(t, x)=\delta \sin p x \sin \omega t
$$

The function (13) satisfies the conditions:

$$
u(0, x)=0, u(t, 0)=0
$$

but does not satisfy the initial condition:

$$
\frac{\partial u}{\partial t}(0, x)=0 \text {. }
$$

We neglect this deviation, because in the cyclic tensile test bar, this condition is not important. In order to satisfy the last boundary condition in (3), the hypothesis that the solution (13) is accept and it satisfies equation (1) in the linear elastic domain, which means that the following relations are satisfied:

$$
p=\frac{\omega}{c}, c^{2}=\frac{E}{\rho}, \omega=2 \pi v
$$

where $v$ is the frequency of traction - compression force. The magnitude of traction expression for $x=l$ and equating to $F_{0}$ is calculated. The expression of the amplitude of solution (13) is obtained. The solution (13) becomes:

$$
u(t, x)=\frac{c F_{0}}{E A_{0} \omega \cos p l} \sin p x \sin \omega t .
$$

It is found that the intensity of the force in $x=0$ is:

$$
F(t, 0)=\frac{F_{0}}{\cos p l} \sin \omega t .
$$

By substituting (17) and (1) in equation (12), and moving all terms to the left member of the equation, we obtained:

$$
F_{0} \sin \omega t-\frac{F_{0}}{\cos p l} \sin \omega t-\int_{0}^{l} \rho A(t, x) \frac{\partial^{2} u(t, x)}{\partial x^{2}} d x=0
$$


It is noted:

$$
R(t, \delta, p)=F_{0} \sin \omega t-\frac{F_{0}}{\cos p l} \sin \omega t-\int_{0}^{l} \rho A(t, x) \frac{\partial^{2} u(t, x)}{\partial x^{2}} d x
$$

the residue of the equation relative to the approximate solution. Approximate solutions where the cross sectional area of the bar varies (partial tear) during the cyclic stretching process is obtained by minimizing the functional (21):

$$
\Omega(\delta)=\int_{0}^{T} R(t, \delta, p) d t \rightarrow \min
$$

Before starting to minimize the functional tests (21), one assumption is made: reducing the cross section of the bar, $A$ can be anywhere between $x=0$ and $x=l$, and strictly in this range. The bar end section remains constant $A_{0}$ being fastened in the jaws of the tensile machine. For this purpose, the function $\varphi(x)$ is chosen as:

$$
\varphi(x)=\beta \sin \frac{\pi x}{l} .
$$

The parameter $\beta$ will vary during the minimization process until they approximately achieve the desired coordinates on a rolling bar Wohler curve. We chose a point which has the coordinate stress point of $574 \mathrm{MPa}$ and 5578 cycles to rupture on the diagram corresponding to the asymmetry coefficient $\mathrm{R}=-1$ for the steel $16 \mathrm{NiCr} 4$, [5].

\section{Results}

The bolt material under virtual fatigue test has the following features:

- circular cylindrical bar diameter $\phi=19 \mathrm{~mm}$ and the length (between the jaws of the tensile machine) $l=49 \mathrm{~mm}$;

- the bar material is the steel $16 \mathrm{NiCr} 4$ with the next basic features: Young modulus, $E=2.1 \cdot 10^{11}$ $\mathrm{N} / \mathrm{m}^{2}$, Poisson ratio 0.28 , stress fatigue limit $\sigma_{R}=157.5 \mathrm{MPa}$, liniar elastic stress limit $\sigma_{e}=210 \mathrm{MPa}$, yield stress limit $\sigma_{c}=800 \mathrm{MPa}$, failure stress limit $\sigma_{r}=900 \mathrm{MPa}$, fatigue strain limit $\varepsilon_{\mathrm{R}}=0.00075$, strain linear elastic limit $\varepsilon_{\mathrm{e}}=0.001$, strain yield limit $\varepsilon_{c}=0.1$, failure strain limit $\varepsilon_{r}=0.1125$;

- the magnitude of the traction force: $F_{0}=162000 \mathrm{~N}$ and the frequency of the traction force, $v=6$ $\mathrm{Hz}$.

A minimal test of correctness of the method of solving the problem was done considering the ideal case of linear elastic bar cyclic stretch. In this case any cross section of the bar area is not affected. For this example $\beta=0$, so for any $x \in[0, l]$, the cross-sectional area remains constant. The values of model parameters $(\beta, \delta, p)$ and maximum values of the relative resultant displacement $u$, and of the strain and stress are given in Table 1.

In the second case the subject is the bar of the same material from which the section effective losses caused by the accumulation of fatigue are taken into account. Note that $\delta$ amplitude parameter at the end to which it applies traction, the pulsation of the function, $\varphi, p$, and maximum values of relative displacement, strain and stress are the same as in the ideal case (Table 1). The number of cycles for the bar hold up to failure, it is different for the two cases. In the ideal case, this number is infinite while the modified model has a finite value (Table 1).

Table 1. Results of numerical experiments (virtual tests)

\begin{tabular}{|l|c|c|c|c|c|r|r|}
\hline \multicolumn{1}{|c|}{ Solution } & $\beta$ & $\delta$ & $p, \mathrm{rad} / \mathrm{s}$ & $u_{\max }, \mathrm{m}$ & $\varepsilon_{\max }$ & $\sigma_{\max , \mathrm{MPa}}$ & cycles \\
\hline $\begin{array}{l}\text { Ideal linear elastic } \\
\text { material }\end{array}$ & 0.00000 & 0.373 & 0.007289 & 0.0001333 & 0.0002721 & 571.4 & $\infty$ \\
\hline $\begin{array}{l}\text { Fatigue sensitive } \\
\text { material }\end{array}$ & 0.00001 & 0.373 & 0.007289 & 0.0001333 & 0.0002721 & 571.4 & 42923 \\
\hline
\end{tabular}


The bar response to the applied cyclic tensile force is given in Fig. 4, 5, 6 and 7. The main feature that differentiates the two models response (ideal linear elastic model that notifies fatigue), is given in Fig. 7. It is shown in Fig. 7 that the area of the critical section decreases approximately in steps, each step corresponding to values exceeding strain and / or stress limit fatigue. Also Fig. 7 shows that in calculating loss of effective section resistance instabilities occur relatively quickly in the calculation (in less than one second by calculating using program [6]).

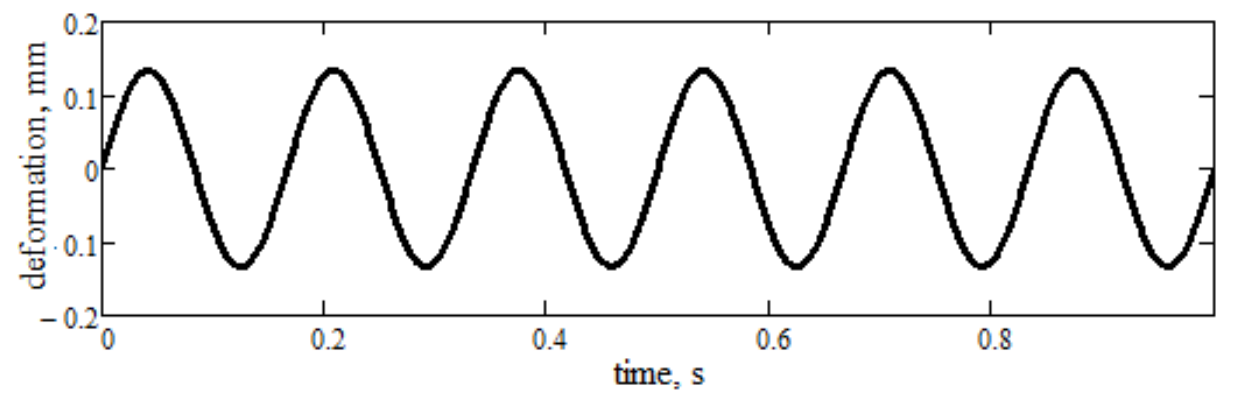

Fig. 4 Deformation (relative resultant displacement) history

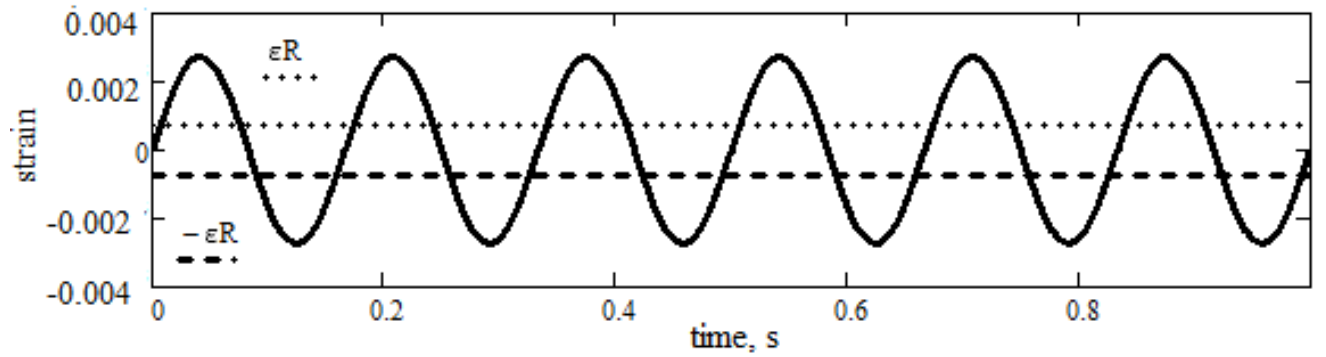

Fig. 5 Strain history

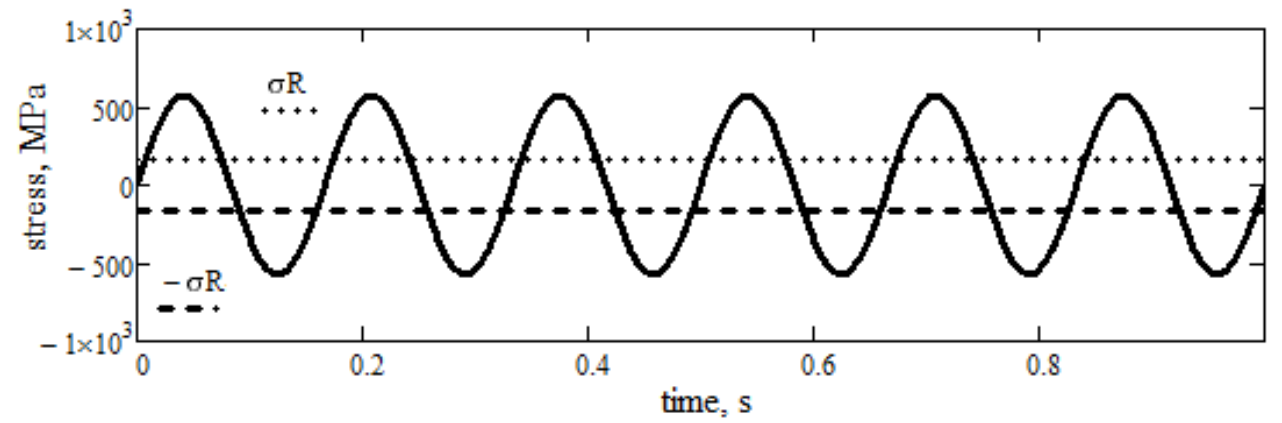

Fig. 6 Stress history

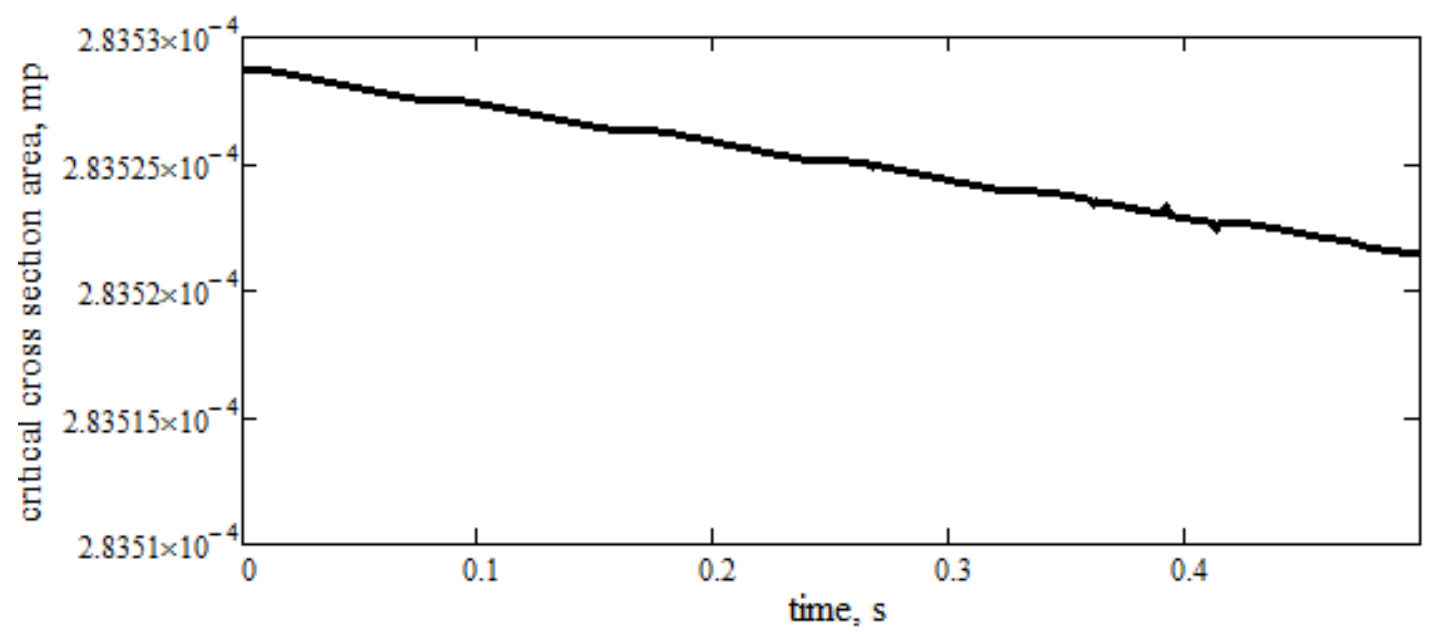

Fig. 7 Critical cross section area history 


\section{Conclusions}

Mathematical modelling of the materials fatigue process is possible using mathematical functions such as those exposed in the nonstandard mathematical models from [2] in this article.

In [2], the indicators of the fatigue intensity were the tensile stress limit and the yield stress limit. Decreasing these parameters of the model could not be observed experimentally in [2]. The indicator of the material fatigue intensity in the mathematical model presented in this paper is the area of the critical section. This area decreases monotonically marking fatigue emphasis. This model provides an explanation for a possible mechanism of fatigue action on the structure. In addition to the [2], experimentally, the mechanism of fatigue installation by decreasing the area of the critical section is shown.

A series of bolts were fatigue tested and the result was constant. The emergency section was located at the bottom of the first turn of the screw thread caught in the nut, located towards the head of the screw. The section where the damage occurred during physical experiments was determined by the existence of a stress concentrator at the bottom turn of the aforementioned thread. The model has two parameters that can come near the fatigue representation of the behaviour of a material with fidelity, compared to concrete material Wohler curve for which the simulator is made. A model raising to this level where it provides a sufficiently accurate Wohler curve, is our goal. In order to achieve a model capable of providing Wohler curve, a concrete material should be addressed, running long (hundreds of thousands and even millions of cycles), issues of stability, convergence and accuracy of mathematical operators are involved.

Another aspect that the model presented in this paper can simulate is due to the fact that the cross section of the bar is variable. Using this advantage, we can simulate the appearance and propagation of cracks within the sections preferably located around the stress concentrator.

For this purpose the initial cross-sectional area, $A_{0}$ will not be constant, but will vary.Achieving these models could lead to savings time and energy, in terms of fatigue test experiments, especially if transformation relations between Wohler curves corresponding to various indices of symmetry exist.

Finally, as in [2], it is stated that the introduction of these models in structural design would be beneficial in relation to current resources, and would lead this to high complexity computation and possibly very large computing times, but for these reasons we are skeptical about the assimilation of these models for the calculation models of the most popular structural analysis software.

\section{References}

[1] Gh. Buzdugan., L. Fetcu, M. Rades.: Mechanical Vibrations. Didactical and Pedagogical Printing House, Bucharest, 1982.

[2] P. Cardei.: Models for Bodies with Unsteady Quality, Revue Roumaine des Sciences Techniques, Serie de Mecanique Appliquee, tome 45 1,Bucharest, 2000, p. 97-109.

[3] http://www.math.uaic.ro/ gani/depozit/EDP.pdf.

[4] V. D., Radulescu.: Partial Differential Equations. Department of Mathematics, University of Craiova 2005, Romania.

[5] Solid Works Material Library, 2012.

[6] Mathcad Professional 2001 Documentation.

[7] Gh. Buzdugan, Strength of Materials, Technical Printing House, Bucharest, 1980.

[8] V. Drobota, Strength of Materials, Didactical and Pedagogical Printing House, 1982. 
[9] A. Wohler, Uber die Festigkeitversuche mit Eisen und Stahl, Zeitschrift fur Bauwesen, Vol. VIII, X, XIII, XVI, and XX, 1860/70, English account of this work is in Engineering, Vol. XI, 1871; As quoted by: Rice C Richard, "SAE Fatigue Design Handbook", $3{ }^{\text {rd }}$ Edition, AE-22, 1997.

[10] N. E. Frost, K. J. Marsh, L. P. Pook, Metal Fatigue, Oxford University Press, 1974.

[11] E. Zahavi, V. Torbilo, Fatigue Design: Life Expectancy of Machine Parts, CRC Press, Inc., 1996.

[12] Stephens I. R., Fatemi A., Stephens R. R., Fuchs H. O., Metal Fatigue in Engineering, John Wiley \& Sons, Inc., 2001. 